\title{
Establishment of an Online Design for Failure Diagnosis through Simulation of the Equivalent Model of $\mathrm{LiMn}_{2} \mathrm{O}_{4}$ Battery
}

\author{
Wenbing Yin ${ }^{1}$, Xihao Tang ${ }^{1}$, Xiao Ma ${ }^{1}$, Danfeng Qiu ${ }^{1, *}$, Gang Bu${ }^{1}$, Yongjun Xia ${ }^{1}$, Bin Zhao ${ }^{2}$, Zixia Lin $^{2}$ and Yi Shi ${ }^{2}$ \\ ${ }^{1}$ Key Laboratory of Radar Imaging and Microwave Photonics (Nanjing Univ. Aeronaut. Astronaut.), Ministry of Education, \\ College of Electronic and Information Engineering, Nanjing University of Aeronautics and Astronautics, Nanjing, China \\ ${ }^{2}$ National Laboratory of Microstructures and School of Electronic Science and Engineering, Nanjing University, Nanjing, China \\ ${ }^{*}$ Corresponding author
}

\begin{abstract}
In electric vehicles (EVs), the lithium-ion battery is used as the main energy storage because of its large capacity and high power density. The traditional battery management system (BMS) simply measures battery voltage and current. Most battery damage is caused by failure of the battery's sensor or actuator error detection, which can affect driving safety. Therefore, the system requires accurate failure diagnosis on the battery is significant. An equivalent circuit model should be established precisely to monitor battery charge, open circuit voltage and to diagnose the possible faults at the same time. In this study, equivalent circuit models of $\mathrm{LiMn}_{2} \mathrm{O}_{4}$ batteries were implemented in the MATLAB Simulink model to determine the limit of voltage and current as well as the method of failure diagnosis. Simulation results indicate the accurate failure diagnosis of the model.
\end{abstract}

Keywords—battery management system; failure diagnosis; state of charge; equivalent circuit model

\section{INTRODUCTION}

In response to the energy crisis and mitigation of global warming, many countries are beginning to attach importance to energy conservation and emission reduction and the development of low-carbon economy[1]. Electricity-driven electric vehicles, carbon dioxide emissions can reduce or even achieve zero emissions, so it has been the concern and rapid development of all countries. However, the cost of the battery is still high[2]. The performance and price of the power battery are the main bottlenecks in the development of electric-driven vehicles [3]. The $\mathrm{LiMn}_{2} \mathrm{O}_{4}$ batteries have become the ideal power source for electric vehicles because of their long life, good safety performance and low cost[4].

To give full play to the dynamic performance of the battery system, improve battery safety, prevent battery charge and discharge, extend battery life, optimize driving performance, and improve the performance of electric vehicles, the battery management system (BMS) should accurately estimate the battery's SOC. SOC is an important parameter to describe the battery charge and discharge capacity. The SOC observer with periodic parameter updates outperforms that without real-time parameter renewal[5]. Therefore, real-time determination of battery parameters is essential for credible SOC estimation.
One of the most critical steps for developing battery prognostics solutions is to establish a battery model that enables the automaker to simulate battery behavior and interpret battery issues in a form understandable by users and designers[6].

Battery is a main component that affects the successful operation of EVs [7]. The proper operation of batteries must be ensured when working. To realize this goal, we need a prevention or diagnosis feature in the BMS for the operation of EVs [8].

In this study, a failure diagnosis system is designed to monitor battery parameters. The equivalent model of the battery is established, and failure diagnosis when the battery incurs all possible faults is simulated using MATLAB Simulink.

\section{ANALYSIS AND MODELING OF LITHIUM-ION BATTERY PARAMETERS}

In this paper, $\mathrm{LiMn}_{2} \mathrm{O}_{4}$ batteries are measured. The $\mathrm{LiMn}_{2} \mathrm{O}_{4}$ cathode material exhibits low cost, safe operation, and low-temperature properties. However, the material is unstable and easily decomposes. This material is mainly used in large and medium-type batteries, such as power batteries.

Table 1 shows some basic parameters of the experimental battery.

TABLE I. BASIC PARAMETERS OF THE EXPERIMENTAL BATTERY

\begin{tabular}{|c|c|}
\hline Rated capacity [Ah] & 30 \\
\hline Nominal voltage $[\mathrm{V}]$ & 3.6 \\
\hline Internal impedance $[\mathrm{m} \Omega]$ & 1.12 \\
\hline Cutoff voltage $[\mathrm{V}]$ & 4.25 \\
\hline Minimum discharge voltage $[\mathrm{V}]$ & 2.4 \\
\hline Maximum discharge current $[\mathrm{A}]$ & $120[4 \mathrm{C}]$ \\
\hline Fast-charge current $[\mathrm{A}]$ & $60[2 \mathrm{C}]$ \\
\hline Working temperature $\left[{ }^{\circ} \mathrm{C}\right]$ & $-20-55$ \\
\hline Cycle life & $\geq 1500$ \\
\hline
\end{tabular}


For the vehicle-mounted power system, the load of the EVs has been changing dynamically so this paper chooses a battery model that can describe the charging and discharging characteristics of the battery--the PNGV battery model[9].

The PNGV battery equivalent model is composed of resistance and capacitance to describe the working characteristics of the battery. This method has clear physical significance and easy identification of model parameters[10]. PNGV model belongs to the low order model, but more than a simple first order battery model, Thevenin model, the RC model of high precision [11], and kinetics of fourth order battery model calculation is too complicated, not easy to use on electric cars.

Considering the influence of temperature on the model parameters, the parameters of PNGV model will be more accurate, thus improving the accuracy of battery SOC estimation. The PNGV model shown in Figure 1 is the standard battery model in the 2001 PNGV battery test manual.

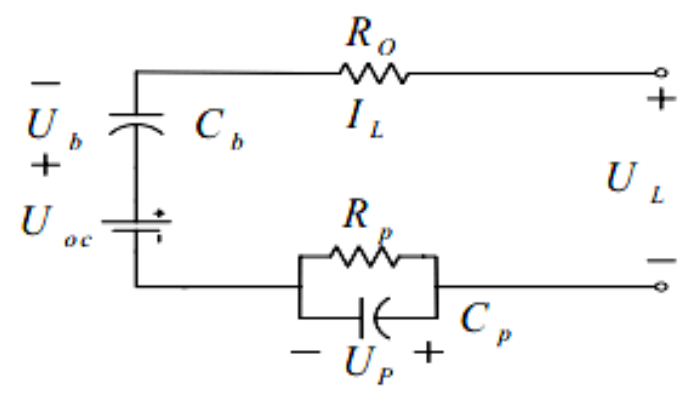

FIGURE I. PNGV EQUIVALENT CIRCUIT MODEL

In the model, $\mathrm{U}_{\text {oc }}$ is the ideal voltage source, indicating the open circuit voltage of the battery; $\mathrm{R}_{0}$ is ohm internal resistance, $R_{p}$ is polarization internal resistance, $C_{p}$ is polarization capacitance. $I_{p}$ is the current on polarization resistance; capacitance $\mathrm{C}_{\mathrm{b}}$ describes the variation of open circuit voltage generated by load current[12].

According to the fundamental theorem of the circuit:

$$
\begin{gathered}
I_{\mathrm{L}}=C \frac{\mathrm{d} U_{\mathrm{p}}}{\mathrm{d} t}+\frac{U_{\mathrm{p}}}{R_{\mathrm{p}}} \\
U_{\mathrm{o}}=I_{\mathrm{L}} R_{\mathrm{o}} \\
U_{\mathrm{L}}=U_{\text {oc }}-U_{\mathrm{o}}-U_{\mathrm{b}}-U_{\mathrm{p}}
\end{gathered}
$$

According to Kirchhoff's voltage law (KVL), the state equation and observation equation of PNGV model is established based on the two capacitance voltage $U_{p}$ and $U_{b}$ in the model, as shown in formula (4)and(5).

$$
\begin{gathered}
{\left[\begin{array}{c}
\dot{U}_{\mathrm{b}} \\
\dot{U}_{\mathrm{p}}
\end{array}\right]=\left[\begin{array}{cc}
0 & 0 \\
0 & -\frac{1}{C_{\mathrm{p}} R_{\mathrm{p}}}
\end{array}\right]\left[\begin{array}{c}
U_{\mathrm{b}} \\
U_{\mathrm{p}}
\end{array}\right]+\left[\begin{array}{c}
\frac{1}{C_{\mathrm{b}}} \\
\frac{1}{C_{\mathrm{p}}}
\end{array}\right] I_{\mathrm{L}}} \\
{\left[U_{\mathrm{L}}\right]=\left[\begin{array}{ll}
-1 & -1
\end{array}\right]\left[\begin{array}{c}
U_{\mathrm{b}} \\
U_{\mathrm{p}}
\end{array}\right]+\left[-R_{\mathrm{o}}\right]\left[I_{\mathrm{L}}\right]+\left[U_{\text {ос }}\right]}
\end{gathered}
$$

The output can be obtained from the state space models and includes SOC, Open Circuit Voltage (OCV), $\mathrm{U}_{\mathrm{b}}$ (voltage difference between the terminal voltage and voltage OCV), and $\mathrm{U}_{\mathrm{p}}[13]$.

In this study, modeling parameters must be determined first. In the experiment, the battery is charged and discharged several times by pulse current, and voltage data are recorded.

During pulse charging and discharging, in addition to changes caused by $\mathrm{R}_{0}, \mathrm{U}_{\mathrm{L}}$ varies because of the polarization effect [14]. The equivalent circuit of the polarization effect is expressed by the $R_{p} C_{p}$ parallel circuit[15]. In the analysis of the polarization effect of lithium battery, continuous values of various parameters of the battery can be obtained using the interpolation algorithm in MATLAB because the polarization parameters are determined from a different SOC. The parameters obtained when the SOC is $50 \%$ are shown in Table 2. The experimental temperature was maintained at $25{ }^{\circ} \mathrm{C}$. The polarization parameters under different SOC were measured.

TABLE II. POLARIZATION PARAmeters In Charging STATE

\begin{tabular}{|c|c|c|}
\hline SOC $[\%]$ & $C_{\mathrm{P}}[\mathrm{F}]$ & $\mathbf{R}_{\mathrm{p}}[\mathrm{m} \Omega]$ \\
\hline 50 & 956.4 & 10.5 \\
\hline
\end{tabular}

\section{DESIgn Of Failure Diagnosis System}

Failure diagnosis determines faults present in a system and time of detection and identify malfunctions in real time as soon and as surely as possible.

Figure 2 shows the block diagram of the designed system. By measuring various parameters, including the current, voltage, OCV and temperature of the battery, the system provides information on the state of the battery and detects the error, then the errors of these anomalies are analyzed, which mainly include the shake elimination, abnormal judgment and conditional filtering[16]. If the fault occurs, the fault information can be separated according to the built-in algorithm, and the failure is stored and managed according to certain format and sequence, and the output control shall be carried out when necessary. 


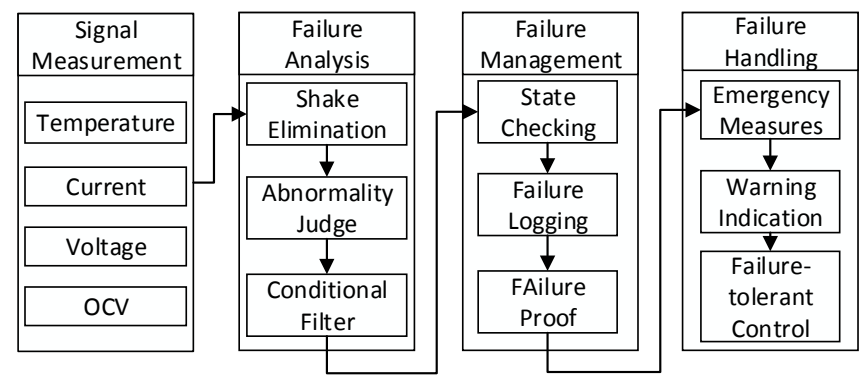

FIGURE II. BLOCK DIAGRAM OF THE FAILURE DIAGNOSIS SYSTEM

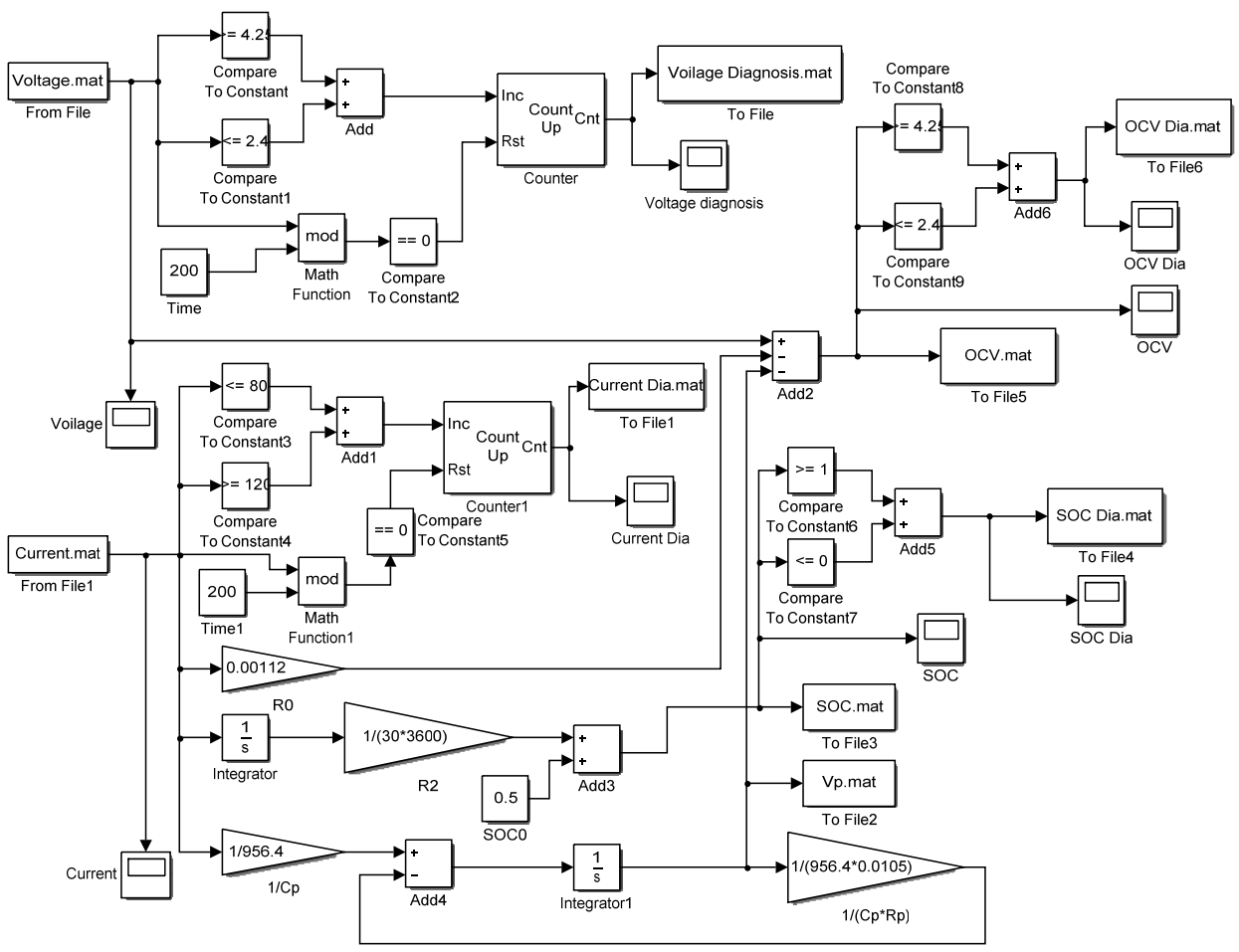

FIGURE III. SIMULINK MODEL OF SIMULATION

Simulation was performed in MATLAB Simulink to determine the working conditions of the system. To simplify the simulation process, we assume a constant temperature of $25{ }^{\circ} \mathrm{C}$ and a SOC of $50 \%$.

Figure 3 shows the failure diagnosis system comprising two inputs, namely, current and voltage, which are related to battery modeling based on PNGV equivalent circuit models. Battery modeling employs parameters $R_{0}, R_{p}, C_{p}$, and $C_{b}$ to measure the outputs SOC and OCV. The output is detected, and the result is recorded in real time. This research focuses on simulation of four outputs (over-current, over-voltage, SOC, and OCV failure diagnosis) to determine the condition of the battery especially under fault condition. When the input voltage and current are given, the system can detect errors at the first attempt.

Current and voltage data are directly obtained from sensor readings on the battery. Based on the characteristics of the battery, the voltage must have a maximum value of $4.25 \mathrm{~V}$ and a minimum value of $2.4 \mathrm{~V}$ to ensure the safety of the battery. The safe current range of the battery should be between $120 \mathrm{~A}$ and $80 \mathrm{~A}$.

The SOC of this model is calculated by Coulomb counting method. We determine the SOC to be $100 \%$ when the voltage reaches the highest maximum value of $4.25 \mathrm{~V}$ or $0 \%$ for the minimum threshold voltage of $2.4 \mathrm{~V}$. OCV is obtained by model simulation according to Equations (4) and (5). The reasonable range is within $2.4 \mathrm{~V}$ and $4.25 \mathrm{~V}$. The simulation results are shown in the following section.

\section{Simulation Result And Analysis}

Voltage and current are added as inputs to the failure diagnosis modeling system to simulate the error status of the battery. Current and voltage signals are assigned with some special values to simulate the error state of the battery pack.

Figure 4(a) shows the input current curve. The output detection is considered to be under the safe condition when the 
current is still in the operation area. As shown in Figure 4(b), when the current is less than $80 \mathrm{~A}$ or more than $120 \mathrm{~A}$, it is regarded as an abnormal value, and the current detection module outputs a value of 1 . If no error occurs, the detector remains at 0 . Then, the error is counted and accumulated. As shown in Figure 4(c), if the number of abnormal values exceeds the set threshold within a unit time, the abnormality is recognized as a fault and an alarm occurs as shown in Figure 4 (d). As shown, the alarm output is 1 , if not, then remains at 0 .

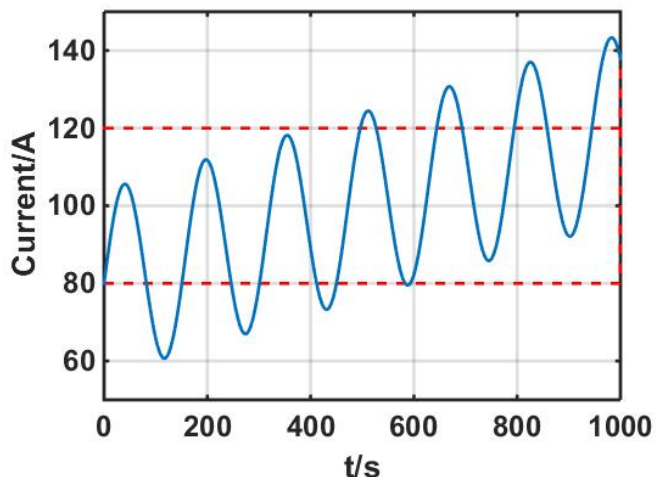

(a)

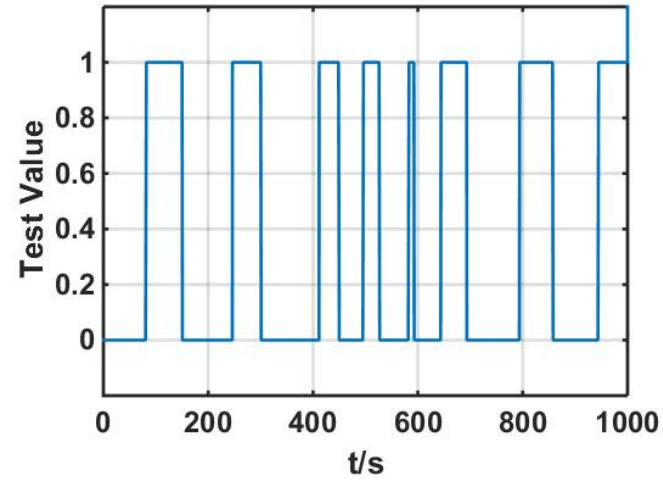

(b)

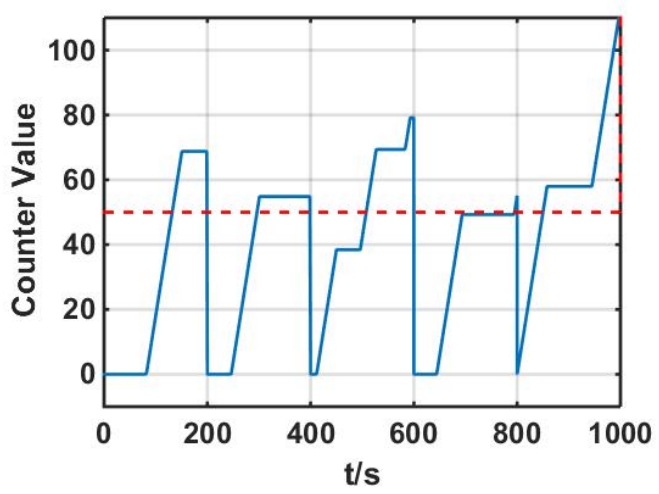

(c)
For a certain fault, if there is no abnormality at present, the value of the counter needs to be accumulated. When the accumulated number reaches a certain level, the fault will be unsealed, and the fault detection again becomes a faultless state as shown in Figure 4(c) shows, the experiment set the value of 200 seconds.

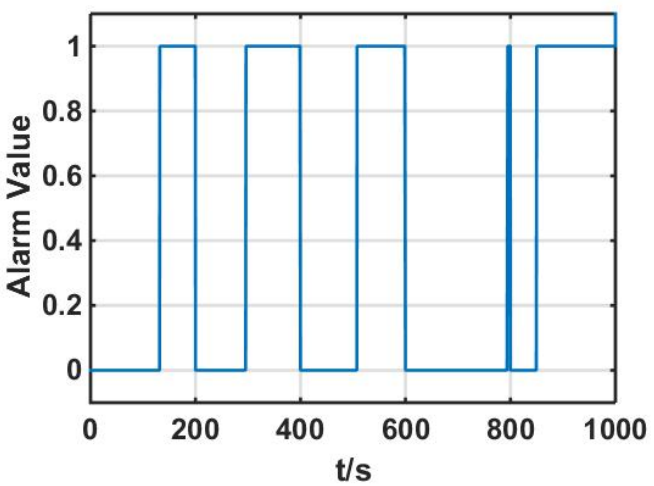

(d)

FIGURE IV. INPUT CURRENT AND CURRENT FAILURE DIAGNOSIS

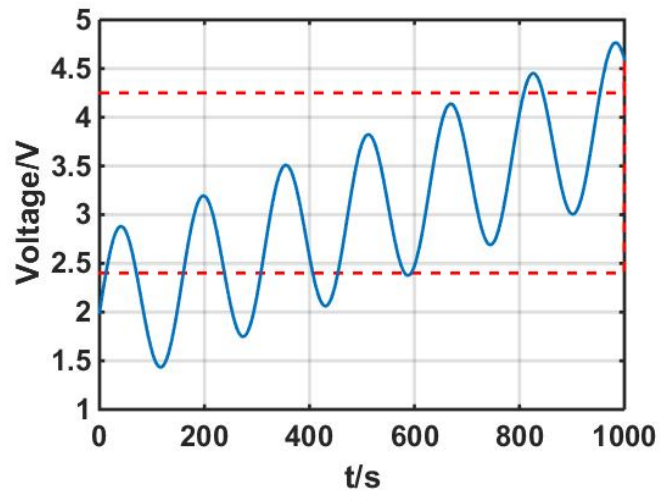

(a)

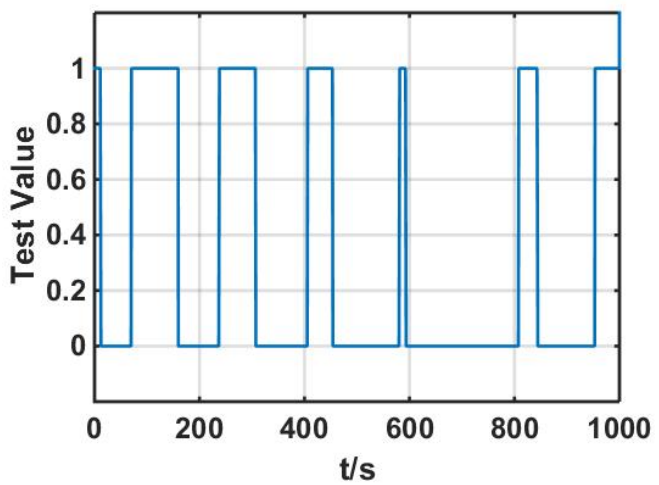


(b)

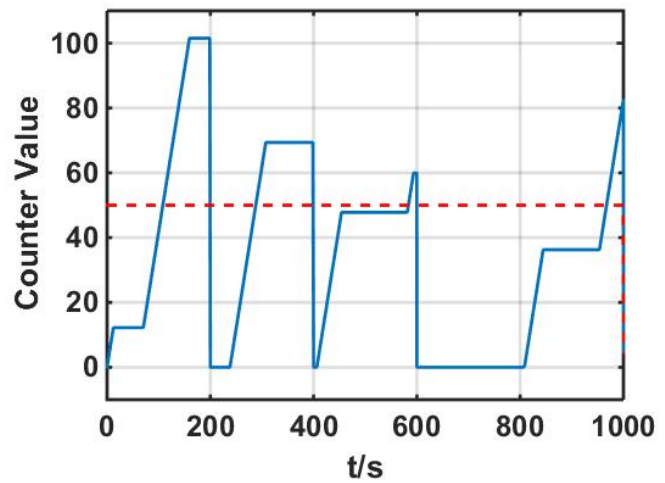

(c)

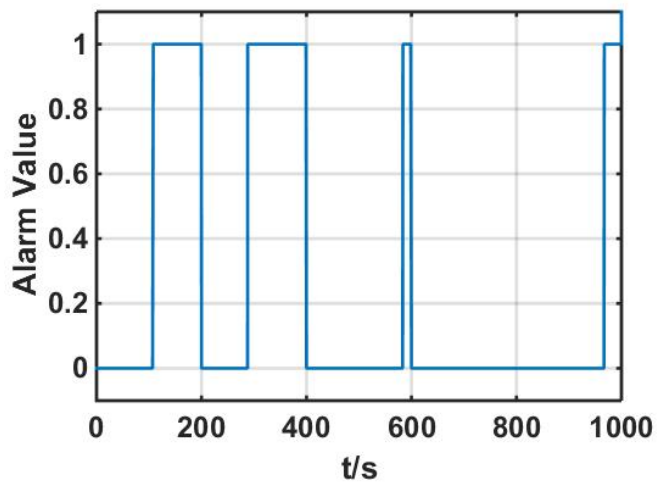

(d)

FIGURE V. INPUT VOLTAGE AND VOLTAGE FAILURE DIAGNOSIS

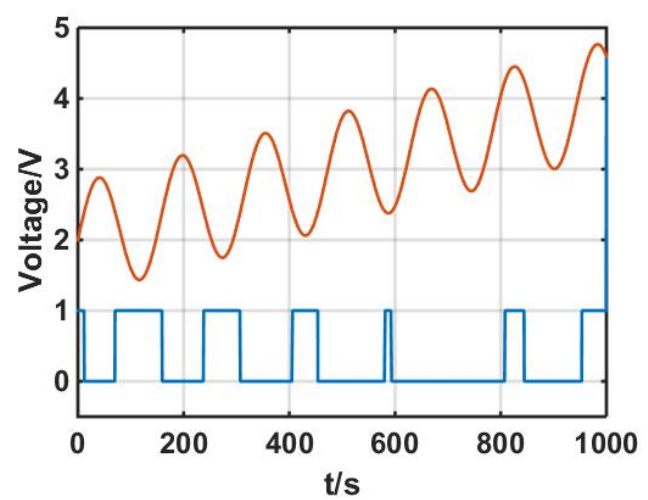

FIGURE VI. OUTPUT OF OCV

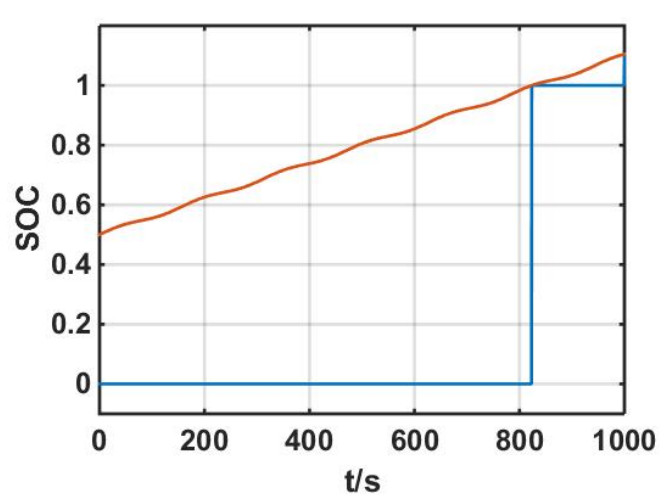

FIGURE VII. OUTPUT OF SOC

Figure 5(a) shows the input voltage curve. The voltage varies from $1.4 \mathrm{~V}$ to $4.8 \mathrm{~V}$. When the voltage becomes greater than $4.25 \mathrm{~V}$ or less than $2.4 \mathrm{~V}$, the detector output value is 1 , as shown in Figure 5(b), indicating that the battery is over-voltage or under-voltage. As shown in Figure 5(c), abnormal values are accumulated, and when the set limit is exceeded, an alarm is generated as shown in Figure 5(d).

The OCV has a maximum value of $4.25 \mathrm{~V}$ and a minimum value of $2.4 \mathrm{~V}$, which are obtained based on the experimental data on the battery before Simulink modeling and failure diagnosis on the battery. Figure 6 shows the simulation results. Again, we can estimate these faults and determine whether they will cause the system to fail.

The SOC of this model is calculated by Coulomb counting method. As shown in Figure 7, a fault is formed even if the SOC is equal to 1 and has a maximum value of $100 \%$ SOC. Hence, when the SOC fault detector reaches a value of 1 , the battery is fully charged or simply runs out of electricity.

\section{CONCLUSION}

In this paper, the equivalent model of the battery is established, and parameters used in the simulation of $\mathrm{LiMn}_{2} \mathrm{O}_{4}$ battery are determined experimentally. Failure diagnosis simulation of voltage and current OCV and SOC are conducted using the given voltage and current values. According to the level of the fault, the system will choose to process it or hand it over to the user and send these fault data to the cloud at the same time. In this way, the user can grasp the condition of the vehicle in time and ensure the safety of driving, manufacturers can also determine the shortcomings of the product from a large amount of data.

\section{ACKNOWLEDGMENT}

This work was supported by the National Natural Science Foundation of China (nos 61471195 and 61404087), the Fundamental Research Funds for the Central Universities (NJ20150017 and NS2014040), and Aeronautical Science Foundation of China (No. 20152052025).

\section{REFERENCES}

[1] L. Lu, X. Han, J. Li, J. Hua, and M. Ouyang, “A review on the key issues for lithium-ion battery management in electric vehicles,”.J. Power Sources 226, 2013, pp. 272-288. 
[2] Hu X, Li S, Peng H. A comparative study of equivalent circuit models for Li-ion batteries. J Power Sources 2012;198:359-67 .

[3] Waag W, Fleischer C, Sauer DU. Critical review of the methods for monitoring of lithium-ion batteries in electric and hybrid vehicles. J Power Sources, 2014; 258:321-39.

[4] Dai H, Guo P, Wei X, Sun Z, Wang J. ANFIS (adaptive neuro-fuzzy inference system) based online SOC (State of Charge) correction considering cell divergence for the EV (electric vehicle) traction batteries. Energy 2015; 80: 350-60.

[5] N. Takami, et al., High-power and long-life Li-ion batteries using lithium titanium oxide anode for automotive and stationary power applications, in:16th International Meeting on Lithium Batteries, ICC Jeju, Korea, 2012.

[6] J. Gao, Y. Zhang, H. He, A real-time joint estimator for model parameters and state of charge of lithium-ion batteries in electric vehicles. Energies. 8 (2015) 8594-612.

[7] Dong G, Wei J, Zhang C, Chen Z. Online state of charge estimation and open circuit voltage hysteresis modeling of $\mathrm{LiFePO} 4$ battery using invariant imbedding method. Appl Energy 2016;162:163-71

[8] A. El Mejdoubi, A. Oukaour, H. Chaoui, Y. Slamani, J. Sabor, and H. Gualous, "Online supercapacitor diagnosis for electric vehicle applications,” IEEE Trans. Veh. Technol., vol. 65, no. 6, pp. 4241-4252, Jun. 2016.

[9] R. German, A. Hammar, R. Lallemand, A. Sari, and P. Venet, "Novel experimental identification method for a supercapacitor multipore model in order to monitor the state of health," IEEE Trans. Power Electron., vol. 31, no. 1, pp. 548-559, Jan. 2016.

[10] Y. Diab, P. Venet, H. Gualous, and G. Rojat, "Self-discharge characterization and modeling of electrochemical capacitor used for power electronics applications,” IEEE Trans. Power Electron., vol. 24, no. 2, pp. 510-517, Feb. 2009.

[11] Feng T, Yang L, Zhao X, Zhang H, Qiang J. Online identification of lithium-ion battery parameters based on an improved equivalent-circuit model and its implementation on battery state-of-power prediction. J Power Sources 2015; 281: 192-203.

[12] He H, Zhang X, Xiong R, Xu Y, Guo H. Online model-based estimation of stateof-charge and open-circuit voltage of lithium-ion batteries in electric vehicles.Energy 2012;39:310-8.

[13] Barre A, Deguilhem B, Grolleau S, G 7 erard M, Suard F, Riu D. A review on lithium-ion battery ageing mechanisms and estimations for automotive applications. J Power Sources 2013; 241: 680-9.

[14] Baronti F, Zamboni W, Femia N, Roncella R, Saletti R. Experimental analysis ofopen-circuit voltage hysteresis in lithium-iron-phosphate batteries. In: Industrial electronics society, IECON 2013-39th annual conference of the IEEE.IEEE; 2013. pp. 6728-33.

[15] Nuhic A, Terzimehic T, Soczka-Guth T, Buchholz M, Dietmayer K. Health diagnosis and remaining useful life prognostics of lithium-ion batteries usingdata-driven methods. J Power Sources 2013; 239: 680-8.

[16] Notten P, Bergveld H, Kruijt W. Battery management systems: design bymodeling. Boston: Kluwer Academic Publisher; 2002. 